\title{
Estimate of Saturation Pressures of Crude Oil by Using Ensemble- Smoother-Assisted Equation of State
}

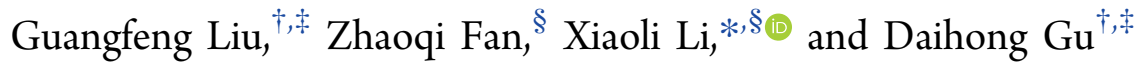 \\ ${ }^{\dagger}$ State Key Laboratory of Petroleum Resources and Prospecting, China University of Petroleum-Beijing, Beijing 102249, China \\ ${ }^{\ddagger}$ Engineering Research Center of Gas Resource Development and Utilization of Ministry of Education, China University of \\ Petroleum-Beijing, Beijing 102249, China \\ ${ }^{\S}$ Department of Chemical and Petroleum Engineering, University of Kansas, Lawrence, Kansas 66045, United States
}

\section{Supporting Information}

\begin{abstract}
The equation of state (EOS) has been extensively used to evaluate the saturation pressures of petroleum fluids. However, the accurate determination of empirical parameters in the EOS is challenging and time-consuming, especially when multiple measurements are involved in the regression process. In this work, an ensemble smoother (ES) -assisted EOS method has been proposed to compute the saturation pressure by intelligently optimizing the to-be-tuned parameters. To be specific, the tobe-tuned parameters for the Peng-Robinson EOS (PR EOS) are integrated into a model input matrix and the measured saturation pressures are collected into a model output matrix. The model input matrix is then integrally and iteratively updated with respect to the model output matrix by using the iterative ES algorithm. For convenience, an in-house module is compiled to implement the ES-assisted EOS for determining the saturation pressures of crude oils. Subsequently, the experimentally measured saturation pressures of 45 mixtures of heavy oil and solvents are used to validate the performance of the in-house module. In addition, 130 measured saturation pressures of worldwide light oil samples are collected to verify the applicability of the developed ES-assisted EOS method. The in-house module is found to be competent by not only matching 45 measured saturation pressures with a better agreement than a commercial simulator but also providing a quantitative means to analyze the uncertainties associated with the estimated model parameters and the saturation pressure. Moreover, the application of the ES-assisted EOS to 130 light oil samples distinctly demonstrates that the new method greatly improves the accuracy and reliability of the EOS regression. Consequently, the in-house module representing the ES-assisted EOS is proven as an efficient and flexible tool to determine the saturation pressure under various conditions and implement uncertain analyses associated with the saturation pressure.
\end{abstract}

\section{INTRODUCTION}

Reservoir fluid properties are the fundamental factors for reserve evaluation, development technique screening, reservoir simulation, and production optimization. It is necessary to understand the interactions among fluids for optimizing labscale or field-scale enhanced oil recovery techniques, for example, $\mathrm{CO}_{2}$ flooding. ${ }^{1,2}$ Saturation pressure of crude oil is one of the reservoir fluid properties that attracts increasing attention in either the academic or the industry since it affects both the operational design and the potential recovery. The saturation pressure is a comprehensive reflection of the composition, critical properties, and other properties of each individual component consisting of the crude oil. ${ }^{3}$ The pressure-volume-temperature (PVT) experiment is the most accurate approach to determine the saturation pressure of reservoir fluid. However, it is impossible to conduct the expensive test for every new fluid sample due to the lengthy procedure and limited resources.

In addition to experiments, theoretical models have been widely used to estimate the crude oil saturation pressure by integrating intrinsic properties of components together with experimentally determined or regressed coefficients. EOS has been developed and extensively applied to quantify fluid properties due to its accuracy and practicability. The accuracy of EOSs and other correlations have been comparatively investigated. ${ }^{3-5}$ It has been demonstrated that either the EOSs or the correlations are powerful to calculate the saturation pressure of the studied crude oil; however, their reliability is limited to a certain degree while applied to crude oils collected from different blocks. It is attributed to the fact that the regression process is usually implemented to derive the to-beestimated coefficients involved in either the EOSs or correlations, and various crude oils intend to yield different values of coefficients. ${ }^{6,7}$ In addition, the regression process could be nonconvergent, provided that the initial guesses of the to-be-estimated coefficients are too far away from its true

Received: October 1, 2018

Revised: November 20, 2018

Accepted: November 20, 2018

Published: November 20, 2018 
values. ${ }^{8}$ Overall, the regression to determine the unknown coefficients is of significant importance to the EOSs.

Numerically, some correlations have been generated based on an abundant database of measured saturation pressures with the assistance of advanced algorithms, for example, gene expression programming., 10 However, these correlations usually present a rough manner to determine the saturation pressure since a large number of measurements of various crude oils are required to obtain reliable and accurate correlations. It is hardly possible to achieve an accurate estimation of the saturation pressure while there is limited data to conduct the regression. However, such advanced algorithms are greatly helpful for estimation of unknown coefficients in EOSs.

The reliability of EOSs determining the saturation pressure is subject to the quality of the to-be-estimated coefficients derived in the regression process, which can be considered as one of history matching problems. As such, the ensemblebased algorithms that have been extensively applied in the history matching process will be a competitive candidate for calibrating the EOSs with limited data. ${ }^{1-17}$ By reproducing the production profiles, the ensemble-based algorithm can effectively estimate permeability, porosity, rock-fluid properties, and other inputs of reservoir simulation. ${ }^{17-22}$ The iterative ensemble smoother (IES), that is, one of the ensemble-based algorithms, has been developed to compute a global update by simultaneously assimilating all available data instead of a sequential data assimilation adopted by the ensemble Kalman filter (EnKF) or the ensemble randomized maximum likelihood algorithm (EnRML). ${ }^{21-24}$ In contrast, the IES algorithm is more efficient because the entire observation is assimilated at once while the data at different time points are assimilated one by one in the sequential data assimilation algorithms. Moreover, the reliability of the sequential data assimilation algorithms might be challenged while handling the EOS equations because of the scatter nature of the PVT property measurements. Therefore, given measured saturation pressure of certain oil samples, the to-be-estimated coefficients in the EOSs can be automatically, coordinately, and efficiently determined with the assistance of the IES algorithm. Also, the uncertainties associated with the to-be-estimated coefficients and its corresponding effects on the estimated saturation pressure can be properly estimated.

In this paper, an ES-assisted EOS is proposed to estimate the saturation pressure of various crude oils. To be specific, the tobe-estimated coefficients of the EOS are included in a matrix that is iteratively tuned and updated by assimilating the measured saturation pressure data. A modified IES algorithm by Fan et al. ${ }^{25}$ is used to dominate the updating process, which directly reflects the influence of various coefficients on the saturation pressure. The ES-assisted EOS has been programmed and applied to estimate the saturation pressure of gas-heavy-oil mixtures. The performance of the ES-assisted EOS is compared with that of the WinProp against the same system. Furthermore, the applicability of the ES-assisted EOS on gas-light-oil mixtures has also been discussed.

\section{METHODOLOGY}

2.1. Peng-Robinson Equations of State (PR EOS). The PR EOS ${ }^{26}$ has been extensively applied to quantify the vaporliquid equilibrium (VLE) properties of reservoir fluids. Therefore, the PR EOS is used as an example to demonstrate the feasibility of the ES-assisted EOS. The PR-EOS is formulated as follows,

$$
\begin{aligned}
p & =\frac{R T}{V_{\mathrm{M}}-b}-\frac{a}{V_{\mathrm{M}}\left(V_{\mathrm{M}}+b\right)+b\left(V_{\mathrm{M}}-b\right)} \\
a & =a_{\mathrm{c}} \alpha\left(T_{\mathrm{r}}, \omega\right) \\
a_{\mathrm{c}} & =0.457235 \frac{R^{2} T_{\mathrm{c}}^{2}}{P_{\mathrm{c}}} \\
\alpha & = \\
& {\left[1+\left(0.37464+1.54226 \omega-0.26992 \omega^{2}\right)\left(1-T_{\mathrm{r}}^{0.5}\right)\right]^{2} }
\end{aligned}
$$

$$
b=0.0777969 \frac{R T_{\mathrm{c}}}{p_{\mathrm{c}}}
$$

where $p$ is pressure in $\mathrm{kPa}, p_{\mathrm{c}}$ is critical pressure in $\mathrm{KPa}, T$ is the temperature in $\mathrm{K}, T_{\mathrm{c}}$ is critical temperature in $\mathrm{K}, T_{\mathrm{r}}$ is reduced temperature, $V_{\mathrm{M}}$ is a molar volume in $\mathrm{m}^{3} / \mathrm{kmol}, \omega$ is the acentric factor, and $R$ is universal gas constant. Regarding mixtures, the parameters $a$ and $b$ are determined by using the van der Waal's mixing rule. ${ }^{27}$

$$
a=\sum_{i} \sum_{j} y_{i} y_{j}\left(a_{i} a_{j}\right)^{0.5}\left(1-\delta_{i j}\right), b=\sum_{j} y_{j} b_{j}
$$

where $y_{i}$ and $y_{j}$ are the compositions of the $i$ th and $j$ th substance, respectively; $\delta_{i j}$ is the binary interaction parameter (BIP) between the $i$ th and $j$ th substance. Numerous efforts have been made to develop BIP correlations for mixtures. ${ }^{28-31}$ In the present work, the BIP values are determined by Gao's correlation $^{29}$ in which the BIP matrix can be regenerated by adjusting only one parameter. The BIP is expressed as a function of critical temperature as follows,

$$
\delta_{i j}=1-\left[\frac{2 \sqrt{T_{\mathrm{ci}} T_{\mathrm{c} j}}}{T_{\mathrm{c} i}+T_{\mathrm{cj}}}\right]^{\theta}
$$

The coefficient $\theta$ can be adjusted to match the experimental data. Then, the properties of a mixture at the equilibrium state can be calculated when the fugacity of all components are identical in both the liquid and the gas phases; that is,

$$
f_{\mathrm{g} i}=f_{\mathrm{li}}
$$

where $f_{g i}$ is the fugacity of component $i$ in the gas phase and $f_{1 i}$ is the fugacity of the same component in the liquid phase.

Given PR EOS, the saturation pressure $\left(p_{\mathrm{s}}\right)$ can be computed by conducting two-phase flash calculation or directly performing saturation pressure calculation; however, both of them are found to be not fully reliable and computationally expensive. ${ }^{32}$ Phase stability examination is a widely accepted approach to determine the phase boundaries. A phase is considered stable if the system has the lowest Gibbs energy indicating there would be no phase splitting, whereas it is considered unstable if the system could have a lower Gibbs energy by splitting into multiple phases. ${ }^{33}$ Michelsen $(1982)^{34}$ proposed phase stability criteria by creating a second phase inside any given mixture to determine the stability. Note that the second phase is possibly vapor-like or liquid-like. Considering its accuracy and efficiency, Michelsen's stability test is applied in this work to search the saturation pressure at a 
given temperature at which the phase stability is changed from unstable to stable status.

2.2. Ensemble Smoother. It is reasonable to consider the PR EOS as a nonlinear system in which the saturation pressure is the output and the compositions, BIPs, and properties of components are the inputs. $\mathrm{Gu}$ and Oliver proposed the EnRML algorithm to handle the history matching problems in strongly nonlinear systems. ${ }^{35}$ Chen and Oliver demonstrated that the EnRML algorithm associated with sequential assimilation was also able to simultaneously incorporate all data to estimate the model parameters. ${ }^{36}$ Therefore, the EnRML-based iterative ensemble smoother was derived, and updating the model parameters, that is, inputs, satisfies the following equation:

$$
\begin{aligned}
m^{l+1}= & \beta_{l} m_{\mathrm{pr}}+\left(1-\beta_{l}\right) m^{l}-\beta_{l} C_{M} G_{l}^{T}\left(C_{D}+G_{l} C_{M} G_{l}^{T}\right)^{-1} \\
& {\left[g\left(m^{l}\right)-p_{\mathrm{smd}}-G_{l}\left(m^{l}-m_{\mathrm{pr}}\right)\right] }
\end{aligned}
$$

where $m_{p r}$ is prior distribution of the ensemble of model parameters; $m^{l}$ and $m^{l+1}$ denotes the ensemble of model parameters at $l$ and $(l+1)$ iterations; $\beta_{l}$ is the damping factor, $0<\beta_{l}<1 ; C_{M}$ is the covariance of model parameters; $G_{l}$ are the sensitivity matrix, which represents the dependence of the model outputs on the model inputs; $C_{D}$ is the covariance of model outputs; $g(\cdot)$ represents the nonlinear system, that is, PR EOS in the present work; $p_{\text {smd }}$ is the measured saturation pressure in $\mathrm{kPa}$. $T$ represents the transpose of a matrix. Fan et al. $^{25}$ proposed modifications to improve the efficiency and accuracy of the iterative ES algorithm by introducing the normalization of model variables and a recursive approach for determining the $\beta_{l}$. The modified iterative ES algorithm is to be used to assist determining the coefficients of EOS in the present work.

Given a series of saturation pressure data of a mixture measured at various temperatures, the implementation of the ES-assisted EOS that is encapsulated in an in-house module is briefly illustrated in a flowchart (see Figure 1). First, the to-betuned parameters of the specific PR EOS for the mixture are selected; meanwhile, the ensemble size and damping factor are initialized. Then the ensemble of inputs $m$ is generated with

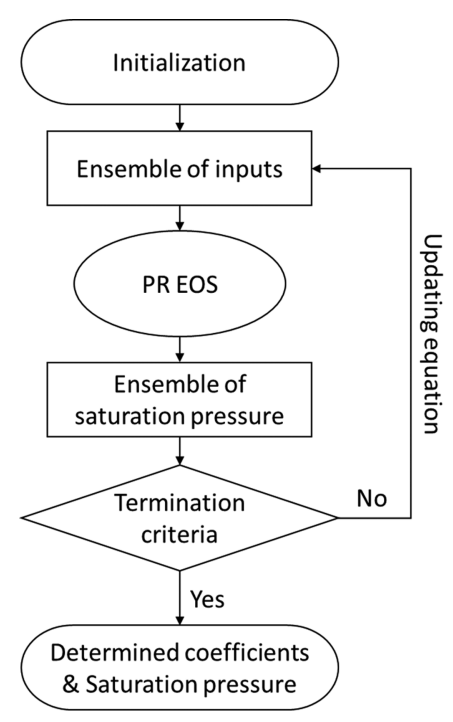

Figure 1. Flowchart for iteratively determining the saturation pressure. the ensemble size and the predefined standard deviation associated with each individual parameter. By applying the PR EOS, the ensemble of saturation pressure is calculated. Subsequently, the ensemble of inputs is regenerated by using the updating equation of the IES algorithm. Note that the $C_{M}$ and $C_{D}$ can be computed from the ensemble of inputs and outputs, respectively. The $G_{l}$ is calculated by $\Delta p_{s}^{l}=G^{l} \Delta m^{l}$ where $\Delta p_{s}^{l}$ represents the difference between the calculated and measured saturation pressure and $\Delta m^{l}$ denotes the difference between the model inputs in two continuous iterations.

To optimize the performance of the ES-assisted EOS program, the necessary termination criteria are proposed to control the iteration. The maximum iteration number of 10 is utilized to restrict the time cost. To avoid invalid iteration, the minimum difference between the model inputs at two continuous iterations is set to be $10^{-3}$ while the minimum difference between the model outputs at the two continuous iterations is set to be $10^{-4}$. The ES-assisted EOS program will terminate once any of these three criteria is satisfied.

\section{EXPERIMENTAL DATA}

3.1. Heavy Oil and Solvents Mixtures. A series of saturation pressure measurements for heavy oil and solvents mixtures have been conducted by using a piston-equipped visual PVT system (PVT-0150-100-200-316-155, DBR, Canada). The heavy oil collected from the Lloydminster area has a molecular weight of $482 \mathrm{~g} / \mathrm{mol}$, a specific gravity of 0.9997 , and a viscosity of $8477.1 \mathrm{cP}$ at the standard condition. The experimental procedure is briefly summarized as follows. The desired amount of oil and solvents are injected into the PVT cell. The temperature of the air bath is set to the desired value for at least $12 \mathrm{~h}$. The continuous depressurization method $^{37}$ is applied in this study. The mixture is depressurized starting from a liquid state at a withdrawal rate of $3.0 \mathrm{~cm}^{3} / \mathrm{h}$. The cell pressure and the volume are recorded during the depressurization process. The saturation pressure can be found from the $p-V$ diagram by locating the turning point. The detailed procedure description can be found elsewhere. ${ }^{38-41}$

$\mathrm{Li}$ et $\mathrm{al}^{38}$ have demonstrated that it is reasonable to characterize the heavy oil as six pseudocomponents in the theoretical calculation. The determined physical and critical properties of six pseudocomponents (i.e., PC1, PC2, PC3, PC4, PC5, and PC6) are listed in Table 1. By considering various compositions, we designed 17 scenarios consisting of 45 experiments where the saturation pressure was separately measured at different temperatures which can be found elsewhere. ${ }^{38-41}$ The composition, temperature, and measured saturation pressure of 45 experiments have been listed in Table 2. These measurements are used to validate the performance of the ES-assisted EOS, and the results will be discussed in section 4.1.

3.2. Light Oil and Solvent Mixtures. The saturation pressures of various oil samples from worldwide fields have been collected to further demonstrate the adaptability of the ES-assisted EOS. Elsharkawy ${ }^{9}$ measured saturation pressures and compositions of 55 mixtures of crude oil samples and solvents (i.e., $\mathrm{N}_{2}, \mathrm{CO}_{2}$, and $\mathrm{H}_{2} \mathrm{~S}$ ) from the Middle East (see experiment No. $1-55$ in Table $S 1$ in the Supporting Information). These experiments cover pressure ranging from $5516 \mathrm{kPa}$ to $34474 \mathrm{kPa}$ and a temperature range of 327.6-394.3 K. These oil samples are featured as the low content of nonhydrocarbons (less than 10\%), oil formation 
Table 1. Properties of Solvents and Six Pseudocomponents Representing the Heavy Oil Sample

\begin{tabular}{|c|c|c|c|c|c|c|}
\hline & critical pressure, $\mathrm{kPa}$ & critical temp, $\mathrm{K}$ & acentric factor & molecular weight & critical molar volume, $\mathrm{m}^{3} / \mathrm{kmol}$ & Rackett parameter \\
\hline $\mathrm{C}_{3}$ & 4246.0 & 369.8 & 0.15 & 44.10 & 0.20 & 0.28 \\
\hline$n \mathrm{C}_{4}$ & 3799.0 & 425.2 & 0.19 & 58.12 & 0.26 & 0.27 \\
\hline $\mathrm{CO}_{2}$ & 7378.0 & 304.1 & 0.22 & 44.01 & 0.09 & 0.27 \\
\hline PC1 & 2048.1 & 669.2 & 0.56 & 171.15 & 0.68 & 0.25 \\
\hline PC2 & 1633.1 & 744.1 & 0.72 & 232.96 & 0.88 & 0.25 \\
\hline PC3 & 1365.0 & 811.4 & 0.88 & 307.85 & 1.07 & 0.24 \\
\hline PC4 & 1136.2 & 894.8 & 1.05 & 436.42 & 1.25 & 0.25 \\
\hline PC5 & 995.9 & 997.2 & 1.19 & 694.52 & 1.37 & 0.27 \\
\hline PC6 & 1066.5 & 1129.6 & 1.20 & 1396.46 & 1.39 & 0.35 \\
\hline
\end{tabular}

Table 2. Measurements of Saturation Pressure Conditioned to Various Compositions and Temperatures

\begin{tabular}{|c|c|c|c|c|c|c|c|c|c|c|c|}
\hline \multirow[b]{2}{*}{ scenario no. } & \multicolumn{9}{|c|}{ composition } & \multirow[b]{2}{*}{ temp, $\mathrm{K}$} & \multirow[b]{2}{*}{ measured $p_{s}, \mathrm{kPa}$} \\
\hline & $\mathrm{C}_{3}$ & $\mathrm{nC}_{4}$ & $\mathrm{CO}_{2}$ & PC1 & PC2 & PC3 & PC4 & PC5 & PC6 & & \\
\hline \multirow[t]{3}{*}{1} & 0.00 & 0.00 & 0.55 & 0.09 & 0.08 & 0.08 & 0.07 & 0.07 & 0.06 & 323.2 & 7942.8 \\
\hline & 0.00 & 0.00 & 0.55 & 0.09 & 0.08 & 0.08 & 0.07 & 0.07 & 0.06 & 343.5 & 9332.5 \\
\hline & 0.00 & 0.00 & 0.55 & 0.09 & 0.08 & 0.08 & 0.07 & 0.07 & 0.06 & 362.6 & 11093.9 \\
\hline \multirow[t]{3}{*}{2} & 0.00 & 0.00 & 0.28 & 0.15 & 0.12 & 0.13 & 0.12 & 0.11 & 0.09 & 324.0 & 3039.0 \\
\hline & 0.00 & 0.00 & 0.28 & 0.15 & 0.12 & 0.13 & 0.12 & 0.11 & 0.09 & 348.4 & 3781.8 \\
\hline & 0.00 & 0.00 & 0.28 & 0.15 & 0.12 & 0.13 & 0.12 & 0.11 & 0.09 & 362.8 & 4212.7 \\
\hline 3 & 0.59 & 0.00 & 0.00 & 0.08 & 0.07 & 0.08 & 0.07 & 0.06 & 0.05 & 323.9 & 1311.7 \\
\hline 4 & 0.73 & 0.00 & 0.00 & 0.05 & 0.05 & 0.05 & 0.04 & 0.04 & 0.04 & 323.9 & 1532.7 \\
\hline 5 & 0.59 & 0.00 & 0.00 & 0.08 & 0.07 & 0.08 & 0.07 & 0.06 & 0.05 & 298.9 & 803.6 \\
\hline 6 & 0.66 & 0.00 & 0.00 & 0.07 & 0.06 & 0.06 & 0.06 & 0.05 & 0.04 & 298.9 & 821.9 \\
\hline 7 & 0.74 & 0.00 & 0.00 & 0.05 & 0.05 & 0.05 & 0.04 & 0.04 & 0.03 & 298.9 & 882.6 \\
\hline \multirow[t]{4}{*}{8} & 0.67 & 0.00 & 0.00 & 0.07 & 0.06 & 0.06 & 0.05 & 0.05 & 0.04 & 298.9 & 867.0 \\
\hline & 0.67 & 0.00 & 0.00 & 0.07 & 0.06 & 0.06 & 0.05 & 0.05 & 0.04 & 323.2 & 1444.0 \\
\hline & 0.67 & 0.00 & 0.00 & 0.07 & 0.06 & 0.06 & 0.05 & 0.05 & 0.04 & 348.2 & 2342.0 \\
\hline & 0.67 & 0.00 & 0.00 & 0.07 & 0.06 & 0.06 & 0.05 & 0.05 & 0.04 & 395.2 & 5030.0 \\
\hline \multirow[t]{4}{*}{9} & 0.00 & 0.62 & 0.00 & 0.08 & 0.07 & 0.07 & 0.06 & 0.05 & 0.05 & 298.9 & 238.8 \\
\hline & 0.00 & 0.62 & 0.00 & 0.08 & 0.07 & 0.07 & 0.06 & 0.05 & 0.05 & 323.2 & 412.3 \\
\hline & 0.00 & 0.62 & 0.00 & 0.08 & 0.07 & 0.07 & 0.06 & 0.05 & 0.05 & 348.2 & 696.2 \\
\hline & 0.00 & 0.62 & 0.00 & 0.08 & 0.07 & 0.07 & 0.06 & 0.05 & 0.05 & 369.2 & 1697.6 \\
\hline \multirow[t]{4}{*}{10} & 0.00 & 0.83 & 0.00 & 0.04 & 0.03 & 0.03 & 0.03 & 0.02 & 0.02 & 298.9 & 247.3 \\
\hline & 0.00 & 0.83 & 0.00 & 0.04 & 0.03 & 0.03 & 0.03 & 0.02 & 0.02 & 323.2 & 454.8 \\
\hline & 0.00 & 0.83 & 0.00 & 0.04 & 0.03 & 0.03 & 0.03 & 0.02 & 0.02 & 348.2 & 828.0 \\
\hline & 0.00 & 0.83 & 0.00 & 0.04 & 0.03 & 0.03 & 0.03 & 0.02 & 0.02 & 369.2 & 2007.8 \\
\hline \multirow[t]{3}{*}{11} & 0.55 & 0.16 & 0.00 & 0.06 & 0.05 & 0.05 & 0.05 & 0.04 & 0.04 & 298.9 & 679.5 \\
\hline & 0.55 & 0.16 & 0.00 & 0.06 & 0.05 & 0.05 & 0.05 & 0.04 & 0.04 & 348.2 & 1816.9 \\
\hline & 0.55 & 0.16 & 0.00 & 0.06 & 0.05 & 0.05 & 0.05 & 0.04 & 0.04 & 396.2 & 4063.0 \\
\hline \multirow[t]{3}{*}{12} & 0.36 & 0.00 & 0.39 & 0.05 & 0.04 & 0.05 & 0.04 & 0.04 & 0.03 & 280.5 & 3173.3 \\
\hline & 0.36 & 0.00 & 0.39 & 0.05 & 0.04 & 0.05 & 0.04 & 0.04 & 0.03 & 298.9 & 4651.1 \\
\hline & 0.36 & 0.00 & 0.39 & 0.05 & 0.04 & 0.05 & 0.04 & 0.04 & 0.03 & 318.8 & 6642.2 \\
\hline \multirow[t]{4}{*}{13} & 0.00 & 0.34 & 0.32 & 0.07 & 0.06 & 0.06 & 0.06 & 0.05 & 0.04 & 298.9 & 3154.0 \\
\hline & 0.00 & 0.34 & 0.32 & 0.07 & 0.06 & 0.06 & 0.06 & 0.05 & 0.04 & 318.8 & 4058.0 \\
\hline & 0.00 & 0.34 & 0.32 & 0.07 & 0.06 & 0.06 & 0.06 & 0.05 & 0.04 & 347.7 & 5670.0 \\
\hline & 0.00 & 0.34 & 0.32 & 0.07 & 0.06 & 0.06 & 0.06 & 0.05 & 0.04 & 391.6 & 8350.0 \\
\hline \multirow[t]{3}{*}{14} & 0.20 & 0.17 & 0.34 & 0.06 & 0.05 & 0.05 & 0.05 & 0.04 & 0.04 & 296.5 & 4867.7 \\
\hline & 0.20 & 0.17 & 0.34 & 0.06 & 0.05 & 0.05 & 0.05 & 0.04 & 0.04 & 317.7 & 6894.8 \\
\hline & 0.20 & 0.17 & 0.34 & 0.06 & 0.05 & 0.05 & 0.05 & 0.04 & 0.04 & 327.9 & 8122.0 \\
\hline \multirow[t]{3}{*}{15} & 0.17 & 0.14 & 0.29 & 0.08 & 0.07 & 0.07 & 0.07 & 0.06 & 0.05 & 302.6 & 4605.7 \\
\hline & 0.17 & 0.14 & 0.29 & 0.08 & 0.07 & 0.07 & 0.07 & 0.06 & 0.05 & 323.9 & 6184.6 \\
\hline & 0.17 & 0.14 & 0.29 & 0.08 & 0.07 & 0.07 & 0.07 & 0.06 & 0.05 & 343.1 & 7839.3 \\
\hline \multirow[t]{3}{*}{16} & 0.15 & 0.13 & 0.25 & 0.10 & 0.08 & 0.08 & 0.08 & 0.07 & 0.06 & 322.1 & 5240.0 \\
\hline & 0.15 & 0.13 & 0.25 & 0.10 & 0.08 & 0.08 & 0.08 & 0.07 & 0.06 & 343.0 & 6591.4 \\
\hline & 0.15 & 0.13 & 0.25 & 0.10 & 0.08 & 0.08 & 0.08 & 0.07 & 0.06 & 362.9 & 8039.3 \\
\hline \multirow[t]{3}{*}{17} & 0.12 & 0.11 & 0.21 & 0.12 & 0.10 & 0.10 & 0.09 & 0.08 & 0.07 & 333.8 & 4764.3 \\
\hline & 0.12 & 0.11 & 0.21 & 0.12 & 0.10 & 0.10 & 0.09 & 0.08 & 0.07 & 353.8 & 5722.6 \\
\hline & 0.12 & 0.11 & 0.21 & 0.12 & 0.10 & 0.10 & 0.09 & 0.08 & 0.07 & 373.4 & 6736.2 \\
\hline
\end{tabular}


factor of $1.0-2.0 \mathrm{~m}^{3} / \mathrm{m}^{3}$, and solution gas-oil ratio of $0-350$ $\mathrm{m}^{3} / \mathrm{m}^{3}$. For theoretical computation, the oil samples have been characterized by $\mathrm{C} 1$ to $\mathrm{C} 6$ and a heptane plus-fraction $\mathrm{C} 7+$. The molecular weight of the $\mathrm{C} 7+$ was determined by a vapor pressure osmometer. More details of the 55 measurements of the Middle East crude oil sample and solvents can be found elsewhere. ${ }^{9}$

In addition, 75 measurements of saturation pressure and compositions of various oil samples from the Middle East, North Sea, and North America have also been collected from the literature to test the validity of the ES-assisted EOS. More details about these 75 measurements can be found from experiment No. 56-130 in Table S1 in Supporting Information.

\section{RESULTS AND DISCUSSION}

4.1. Validation: Heavy Oil Data. Although the saturation pressure of a crude oil is affected by multiple factors, only the BIP coefficients of $\mathrm{CO}_{2}$-hydrocarbon and hydrocarbonhydrocarbon $(\mathrm{HC}-\mathrm{HC})$ together with the critical pressure, critical temperature, and acentric factor of the heptane plusfraction are tuned in the present work to match the 45 measured saturation pressures. Considering the trade-off between the accuracy and the computational cost, an ensemble size of 50 and an initial damping factor of 0.5 are used in the developed in-house module. By assigning Gauss-distributed errors to the to-be-estimated parameters, an input matrix is generated with a size of $5 \times 50$ and includes various configurations of BIP coefficients, critical pressure, critical temperature, and acentric factor of the heptane plus-fraction. Moreover, Gaussian distributed errors are also added to the 45 measured saturation pressure, which is anticipated to improve the performance of the ensemble-based algorithm to a certain degree. $^{42}$ Subsequently, the input matrix is iteratively updated by using eq 9 .

Scenarios 1 and 2 in Table 2 are used as examples to demonstrate the performance of the ES-assisted EOS. Figure $2 \mathrm{a}$ presents the distribution of the initial ensemble of saturation pressure calculated by using the initial ensemble of model inputs. It is worthwhile noting that the box plot in Figure 2 includes 95th percentile (cross mark at the top), 75th percentile (top of the box), mean (square symbol), 50th percentile (bar in the box), 25th percentile (bottom of the box), and fifth percentile (cross mark at the bottom). Those box plots directly and quantitatively represent the uncertainties of the calculated saturation pressure inherited from the uncertainties associated with the model inputs. After any of the aforementioned termination criteria is satisfied, the final ensemble of saturation pressure is obtained and shown in Figure $2 \mathrm{~b}$. It can be found that the saturation pressure distribution of most experiments has converged to its measured one to a large extent. Experiment no. 1 is relatively overcorrect by the updated model inputs, which is unavoidable while matching multiple measurements simultaneously.

An ensemble of estimated model inputs has also been obtained and shown in Figure 2c, which is with respect to the saturation pressure in Figure $2 \mathrm{~b}$. The uncertainties indicated by the box plot demonstrate that the critical pressure, critical temperature, and acentric factor of the heptane plus-fraction is less important to the saturation pressure evaluation compared to the BIP coefficients. The quantified uncertainties associated with the model inputs provide more value options for analyzing the fluid properties in a large-scale oil field. In

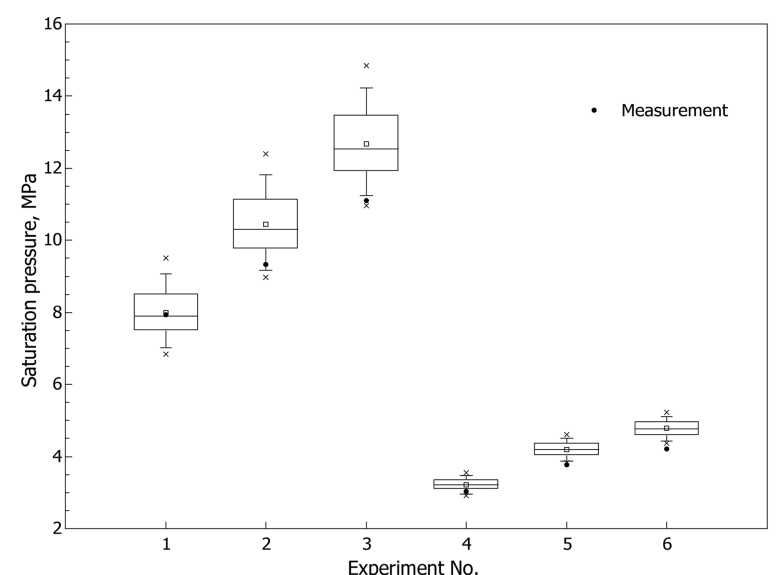

(a)

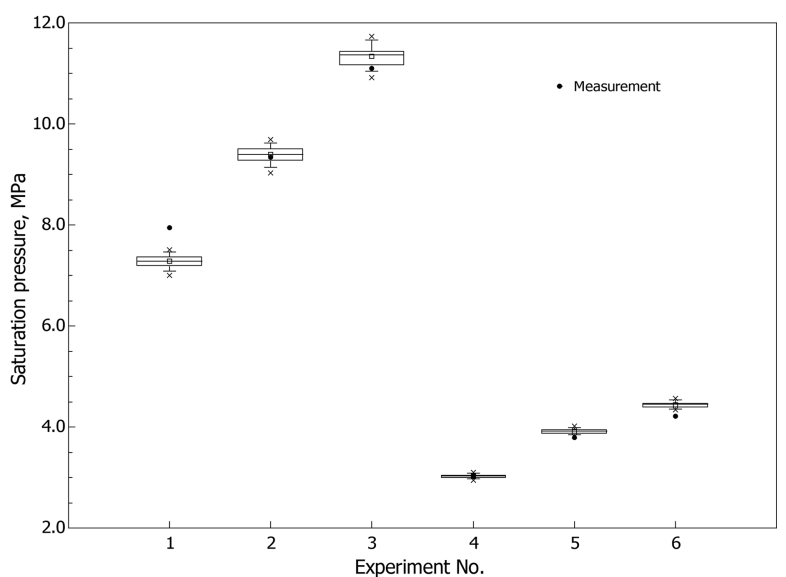

(b)

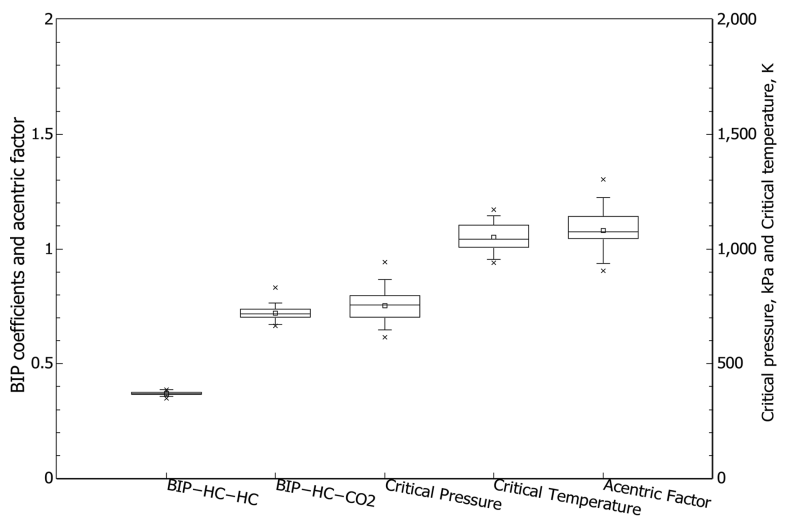

(c)

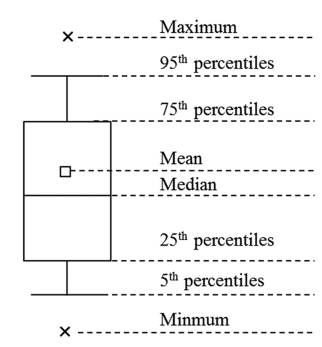

(d)

Figure 2. (a) Initial ensemble and (b) final ensemble of saturation pressure obtained by ES-assisted EOS; (c) estimated model inputs; (d) schematic of the boxes indicating the uncertainty. 
addition, the possible saturation pressure indicated by the box plot conveys more valuable information to the economic analysis, and therefore, is especially conducive to optimize the saturation-pressure-based operational parameters at the field scale.

4.2. ES-Assisted EOS versus WinProp. The calculation results of the ES-assisted EOS are compared with that of the CMG WinProp module, which is an extensively used tool to determine the saturation pressure of petroleum fluids. Both the ES-assisted EOS and the WinProp are employed to estimate the saturation pressures of all 45 experiments in section 3.1 by using the same model inputs. Table 3 shows the initial guesses

Table 3. Tuned Parameters by ES-Assisted EOS and WinProp

\begin{tabular}{llcc}
\multicolumn{1}{c}{ tuned parameters } & $\begin{array}{c}\text { initial } \\
\text { value }\end{array}$ & $\begin{array}{c}\text { estimated by ES- } \\
\text { assisted EOS }\end{array}$ & $\begin{array}{c}\text { estimated by } \\
\text { WinProp }\end{array}$ \\
$\begin{array}{c}\text { critical pressure of } \mathrm{C}+, \\
\text { kPa }\end{array}$ & 1066.5 & 1582.0 & 1032.8 \\
critical temp of $\mathrm{C} 7+, \mathrm{K}$ & 1129.6 & 718.0 & 903.7 \\
acentric factor of $\mathrm{C} 7+$ & 1.198 & 1.565 & 1.322 \\
$\mathrm{HC}-\mathrm{HC}$ interaction & 1.000 & 0.619 & 1.068 \\
coefficient & & & \\
BIP: $\mathrm{CO}_{2}-\mathrm{C}_{3}$ & & 0.135 & 0.125 \\
BIP: $\mathrm{CO}_{2}-n \mathrm{C}_{4}$ & & 0.130 & 0.115 \\
BIP: $\mathrm{CO}_{2}-\mathrm{PC} 1$ & 0.076 & 0.105 \\
BIP: $\mathrm{CO}_{2}-\mathrm{PC} 2$ & & 0.097 & 0.143 \\
BIP: $\mathrm{CO}_{2}-\mathrm{PC} 3$ & 0.114 & 0.173 \\
BIP: $\mathrm{CO}_{2}-\mathrm{PC} 4$ & & 0.136 & 0.000 \\
BIP: $\mathrm{CO}_{2}-\mathrm{PC} 5$ & 0.161 & 0.000 \\
BIP: $\mathrm{CO}_{2}-\mathrm{PC} 6$ & 0.091 & 0.200 \\
\hline
\end{tabular}

and the estimation of model inputs by the ES-assisted EOS and the WinProp, respectively. Since the WinProp can only tune the BIPs between $\mathrm{CO}_{2}$ and individual hydrocarbons instead of the interaction coefficients of $\mathrm{CO}_{2}-$ hydrocarbon, the BIPs for various pairs of $\mathrm{CO}_{2}$ and hydrocarbon are listed. The variation between results obtained by the ES-assisted EOS and the WinProp distinctly demonstrate that there are uncertainties associated with the EOSs. Therefore, the quantification of the uncertainty by the ES-assisted EOS is of great importance to the applicability of the EOSs in practice.

Figure 3 presents the comparison of two methods in both qualitative and quantitative manners. The diagonal black line represents the perfect match between the calculated and the measured saturation pressure. It can be observed that the solid squares are closer to the perfect match line while the solid circles are more scattered. Quantitatively, regressions have been made to the ES-assisted EOS and WinProp results, respectively. The ES-assisted EOS yields a coefficient of determination of 0.85 while the WinProp gives a 0.78 . This implies that the EOS is more powerful in simultaneously estimating the saturation pressures of multiple experiments than the WinProp. In addition, the performance of the ESassisted EOS is rarely affected by the initial guesses of model inputs since the ES-assisted EOS can intelligently search for the optimal solution regardless of the initial guess. In contrast, the accuracy of the WinProp is dramatically impacted by the initial guesses of the model inputs. An abnormal result will be generated by the WinProp provided that inappropriate model inputs are used. In other words, the ES-assisted EOS is not only more accurate but also more flexible in handling the saturation pressure estimation.

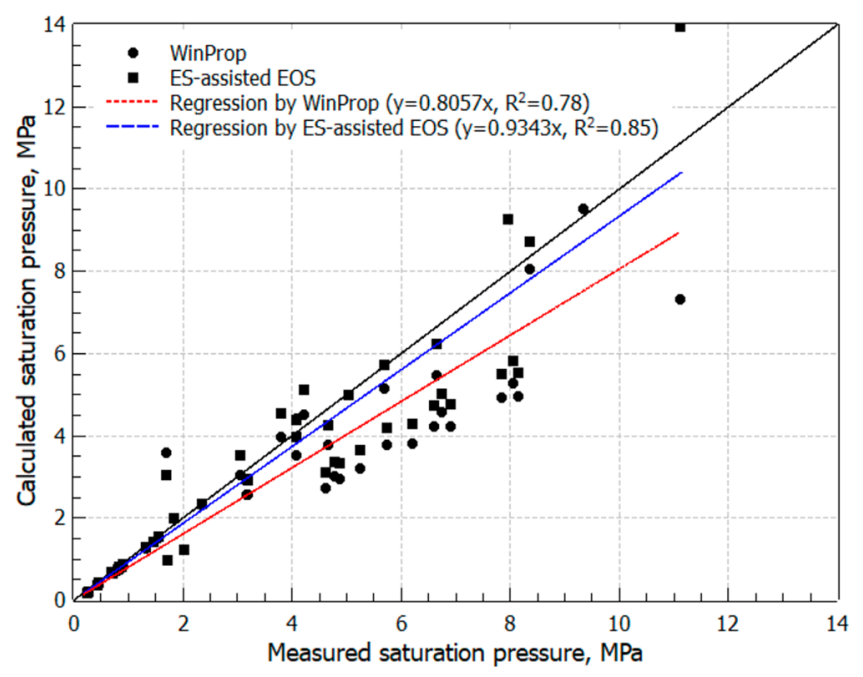

Figure 3. Comparison of ES-assisted EOS and WinProp in the estimation of saturation pressures of 45 experiments.

4.3. Adaptability of the ES-Assisted EOS for Light Oil. The 55 measurements of Middle East oil samples and the other 75 measurements are characterized by the ES-assisted EOS, respectively. The to-be-tuned parameters for estimating the saturation pressure for mixtures of light oil and solvents include BIP coefficients for different pairs of gas components. To compare the performance of the ES-assisted EOS and other methods, cumulative errors of different methods are calculated by summarizing the relative error of each calculation corresponding to one experimental point.

As can be seen in Figure 4, the cumulative error of the ESassisted EOS method is distinctly smaller than that of Soave-

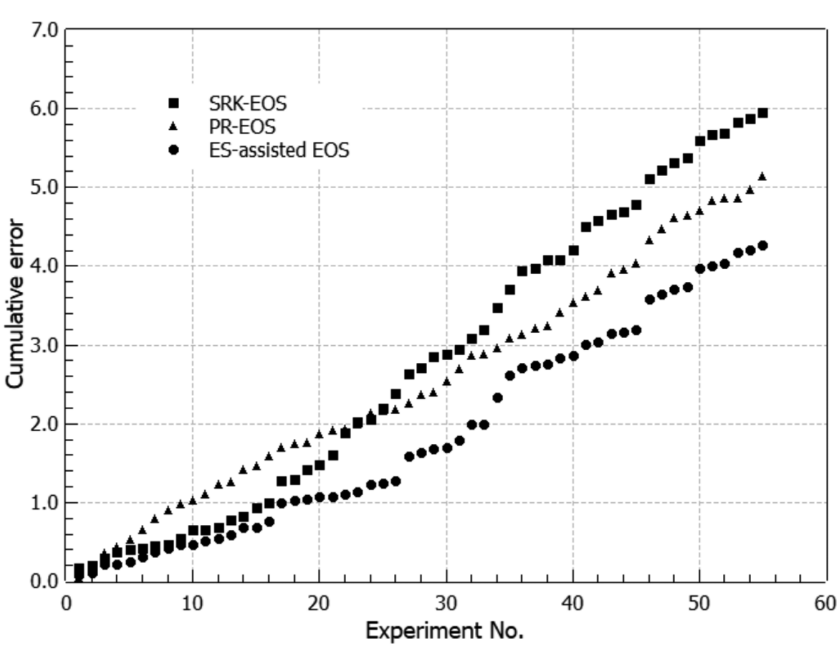

Figure 4. Cumulative error of various methods for matching the 55 measurements of light oil samples from the Middle East.

Redlich-Kwong (SRK) EOS and PR-EOS methods calculated by Elsharkawy. ${ }^{.}$Quantitatively, the ES-assisted EOR yields an average absolute relative deviation (AARD) of $7.7 \%$ while the SRK EOS and PR-EOS are associated with AARDs of $10.8 \%$ and $9.4 \%$, respectively. The low AARD generated by the ESassisted EOR method demonstrate its adaptability in dealing with light oil samples and its superiority over the other two methods. We also apply the ES-assisted EOS to match the saturation pressure of 75 worldwide light oil samples as 
aforementioned. The cumulative errors of different methods for these 75 measurements have been shown in Figure 5 .

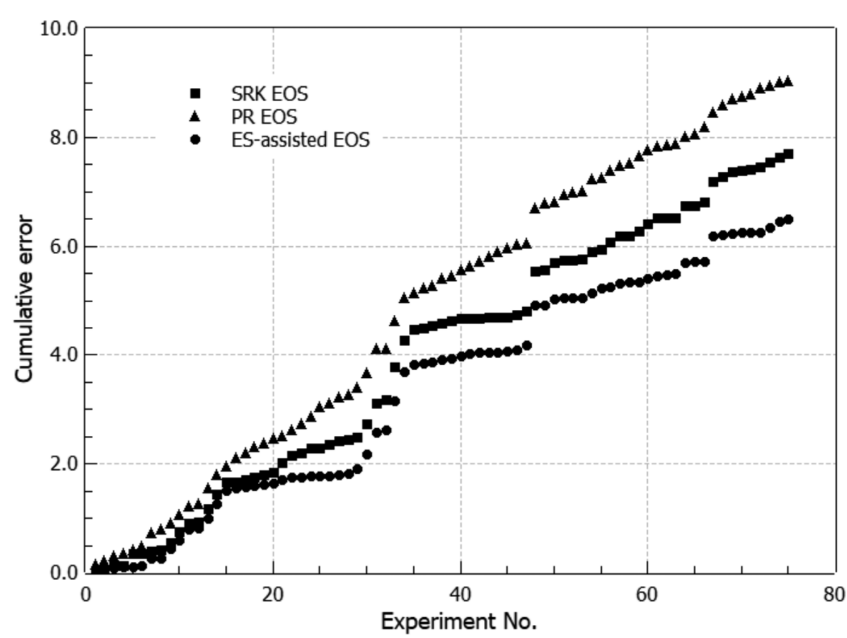

Figure 5. Cumulative error of various methods for matching the 75 measurements of worldwide light oil samples.

Similarly, the ES-assisted EOS method dramatically exceeds the other two methods with the lowest AARD of $8.6 \%$. The AARDs of the SRK-EOS and PR-EOS methods are $12.1 \%$ and $10.3 \%$, respectively. These two applications of the ES-assisted EOS method indicate its feasibility and flexibility in handling different oil samples. More demonstrations can be conducted by estimating more model inputs with sufficient measurement of the fluid properties.

\section{CONCLUSIONS}

An in-house module to implement the ES-assisted EOS has been developed to estimate the saturation pressure of various petroleum fluids. The validity of the in-house module has been well proven by successfully matched experimental measured 45 saturation pressures in the laboratory. The ES-assisted EOS comprises important progress to the applicability of the EOSs since it can automatically optimize the model's parameters or coefficients of the EOSs and provide an explicit way to analyze the uncertainties associated with the estimated saturation pressure. The validation against the measured saturation pressure of heavy oil samples demonstrates that the ESassisted EOS is capable of matching the measured saturation pressure with determined model inputs. The developed ESassisted EOS avoids the possible collapse of the regression process caused by inappropriate initial guesses of model inputs. It is attributed to the fact that the ES-assisted EOS can intelligently search for the optimal solution regardless of the initial guess. The quantified uncertainties associated with the model inputs provide more value options for analyzing the fluid properties in a large-scale oil field, while the possible saturation pressured indicated by the box plot conveys more valuable information to the economic analysis.

In addition, the application of the ES-assisted EOS to extensive worldwide light oil samples indicates its adaptability in various oil samples. Consequently, the in-house module representing the ES-assisted EOS can be an efficient and flexible tool to determine the saturation pressure under various conditions and implement uncertain analyses associated with the saturation pressure, which is of the essence to oil recovery. It is worthwhile noting that the ES-assisted EOS method is conveniently applied to other EOSs for intelligently updating or estimating the to-be-tuned parameters, which then improves the accuracy and reliability of those EOSs.

\section{ASSOCIATED CONTENT}

\section{Supporting Information}

The Supporting Information is available free of charge on the ACS Publications website at DOI: 10.1021/acs.iecr.8b04812.

Composition, specific gravity, molecular weight, temperature, measured and calculated saturation pressure of 130 crude oil samples (PDF)

\section{AUTHOR INFORMATION}

\section{Corresponding Author}

*Tel.: 1-785-864-3209. Email: li.xiaoli@ku.edu. ORCID

\section{Xiaoli Li: 0000-0003-2989-1583}

\section{Notes}

The authors declare no competing financial interest.

\section{ACKNOWLEDGMENTS}

The authors acknowledge the National Natural Science Foundation of China (No. 51404282) to G. Liu for the financial support. The authors acknowledge a Start-up fund and a New Faculty General Research fund to X. Li from the University of Kansas.

\section{REFERENCES}

(1) Zhou, X.; Yuan, Q.; Zhang, Y.; Wang, H.; Zeng, F.; Zhang, L. Performance Evaluation of $\mathrm{CO}_{2}$ Flooding Process in Tight Oil Reservoir via Experimental And Numerical Simulation Studies. Fuel 2019, 236, 730-746.

(2) Zhou, X.; Yuan, Q.; Peng, X.; Zeng, F.; Zhang, L. A Critical Review of The $\mathrm{CO}_{2}$ Huff- $n$-Puff Process for Enhanced Heavy Oil Recovery. Fuel 2018, 215, 813-824.

(3) Wu, R.; Rosenegger, L. Comparison of PVT Properties from Equation of State Analysis and PVT Correlations for Reservoir Studies. J. Can. Pet. Technol. 2000, 39, 45-51.

(4) Ahmed, T. Comparative Study of Eight Equations of State for Predicting Hydrocarbon Volumetric Phase Behavior. SPE Reservoir Eng. 1988, 3 (1), 337-348.

(5) Danesh, A.; Xu, D. H.; Todd, A. C. Comparative Study of Cubic Equations of State for Predicting Phase Behaviour And Volumetric Properties of Injection Gas-Reservoir Oil Systems. Fluid Phase Equilib. 1991, 63 (3), 259-278.

(6) Whitson, C. H. Characterizing the Hydrocarbon Plus Fraction. SPEJ, Soc. Pet. Eng. J. 1983, 23 (4), 683-694.

(7) Coats, K. H.; Smart, G. T. Application of a Regression-Base EOS PVT Program to Laboratory Data. SPE Reservoir Eng. 1986, 1 (3), 277-299.

(8) Baker, L. E.; Luks, K. D. Critical Point and Saturation Pressure Calculations for Multipoint Systems. SPEJ, Soc. Pet. Eng. J. 1980, 20 (1), 15-24.

(9) Elsharkawy, A. M. An Empirical Model for Estimating the Saturation Pressures of Crude Oils. J. Pet. Sci. Eng. 2003, 38 (1-2), $57-77$.

(10) Ahmadi, M. A.; Zendehboudi, S.; James, L. A.; Elkamel, A.; Dusseault, M.; Chatzis, I.; Lohi, A. New Tools to Determine Bubble Point Pressure of Crude Oils: Experimental And Modeling Study. J. Pet. Sci. Eng. 2014, 123, 207-216.

(11) Houtekamer, P. L.; Mitchell, H. L. Data Assimilation Using an Ensemble Kalman Filter Technique. Mon. Weather Rev. 1998, 126 (3), 796-811. 
(12) Evensen, G. The Ensemble Kalman Filter: Theoretical Formulation and Practical Implementation. Ocean Dynam. 2003, 53, 343-367.

(13) Gu, Y.; Oliver, D. S. History Matching of the PUNQS3 Reservoir Model Using the Ensemble Kalman Filter. SPE J. 2005, 10 (2), 51-65.

(14) Zhang, Y.; Li, H.; Yang, D. Simultaneous Estimation of Relative Permeability and Capillary Pressure Using Ensemble-based History Matching Techniques. Transp. Porous Media 2012, 94 (1), 259-276.

(15) Zhang, Y.; Yang, D. Simultaneous Estimation of Relative Permeability and Capillary Pressure for Tight Formations Using Ensemble-Based History Matching Method. Comput. Fluids 2013, 71 (1), 446-460.

(16) Fan, Z.; Zhang, Y.; Yang, D. Estimation of Three-Phase Relative Permeabilities for a Water-Alternating-Gas Process by Use of an Improved Ensemble Randomized Maximum-Likelihood Algorithm. SPE Reservoir Eval. Eng. 2016, 19 (4), 683-693.

(17) Zhang, Y.; Fan, Z.; Yang, D.; Li, H.; Patil, S. Simultaneous Estimation of Relative Permeability and Capillary Pressure for PUNQ-S3Model with A Damped Iterative-Ensemble-Kalman-Filter Technique. SPE J. 2017, 22 (3), 971-984.

(18) Wen, X. H.; Chen, W. H. Real-Time Reservoir Model Updating Using Ensemble Kalman Filter. SPE J. 2006, 11 (4), 431-442.

(19) Wen, X. H.; Chen, W. H. Some Practical Issues on Real-time Reservoir Model Updating Using Ensemble Kalman Filter. SPE J. 2007, 12 (2), 156-166.

(20) Oliver, D. S.; Chen, Y. Recent Progress on Reservoir History Matching: A Review. Computat. Geosci. 2011, 15 (1), 185-221.

(21) Fan, Z.; Yang, D. Determination of Three-Phase Relative Permeability in CHOPS Processes by Use of an Improved Ensemble Smoother. Proceedings of the SPE Reservoir Characterisation and Simulation Conference, Abu Dhabi, UAE, May 8-10, 2017.

(22) Fan, Z.; Yang, D. Estimation of Three-phase Relative Permeabilities for A WAG Process Using An Improved Ensemble Randomized Maximum Likelihood Algorithm. Proceedings of the SPE Reservoir Characterisation and Simulation Conference, Abu Dhabi, UAE, May 8-10, 2017.

(23) Van Leeuwen, P. J.; Evensen, G. Data Assimilation and Inverse Methods in Terms of a Probabilistic Formulation. Mon. Weather Rev. 1996, 124, 2898-2913.

(24) Skjervheim, J.; Evensen, G. An Ensemble Smoother for Assisted History Matching. Paper SPE 141929. Proc. SPE Reservoir Simul. Symp. 2011, No. 141929.

(25) Fan, Z.; Yang, D.; Chai, D.; Li, X. Estimation of Relative Permeability and Capillary Pressure Using a Modified Iterative Ensemble Smoother. J. Energy Resour. Technol. 2019, 141 (2), 022901-022901-9.

(26) Peng, D. Y.; Robinson, D. B. A New Two-constant Equation of State. Ind. Eng. Chem. Fundam. 1976, 15 (1), 59-64.

(27) Edmister, W. C.; Lee, B. I. Applied Hydrocarbon Thermodynamics, 2nd ed.; Gulf Publishing: Houston, TX, 1984.

(28) Chueh, P. L.; Prausnitz, J. M. Vapor-Liquid Equilibria at High Pressures: Calculation of Partial Molar Volumes in Non Polar Liquid Mixtures. AIChE J. 1967, 13 (6), 1099-1107.

(29) Gao, G.; Daridon, J. L.; Saint-Guirons, H.; Xans, P.; Montel, F. A Simple Correlation to Evaluate Binary Interaction Parameters of the Peng-Robinson Equation of State: Binary Light Hydrocarbon Systems. Fluid Phase Equilib. 1992, 74 (15), 85-93.

(30) Kordas, A.; Tsoutsouras, K.; Stamataki, S.; Tassios, D. A Generalized Correlation for the Interaction Coefficients of $\mathrm{CO}_{2}$ Hydrocarbon Binary Mixtures. Fluid Phase Equilib. 1994, 93, 141166.

(31) Moysan, J. M.; Paradowski, H.; Vidal, J. Prediction of Phase Behaviour of Gas-Containing Systems With Cubic Equations of State. Chem. Eng. Sci. 1986, 41 (8), 2069-2074.

(32) Whitson, C. H.; Brulé, M. R. Phase Behavior; Henry, L. Doherty Memorial Fund of AIME, Society of Petroleum Engineers: Richardson, TX, 2000.
(33) Baker, L. E.; Pierce, A. C.; Luks, K. D. Gibbs Energy Analysis of Phase Equilibria. SPEJ, Soc. Pet. Eng. J. 1982, 22 (5), 731-742.

(34) Michelsen, M. L. The Isothermal Flash Problem. Part I. Stability. Fluid Phase Equilib. 1982, 9 (1), 1-19.

(35) Gu, Y.; Oliver, D. S. An Iterative Ensemble Kalman Filter for Multiphase Fluid Flow Data Assimilation. SPE J. 2007, 12 (4), 438446.

(36) Chen, Y.; Oliver, D. S. Ensemble randomized maximum likelihood method as an iterative ensemble smoother. Math. Geosci. 2012, 44 (1), 1-26.

(37) Badamchi-Zadeh, A.; Yarranton, H. W.; Svrcek, W. Y.; Maini, B. B. Phase Behaviour and Physical Property Measurements for VAPEX Solvents: Part I. Propane and Athabasca Bitumen. J. Can. Pet. Technol. 2009, 48 (1), 54-61.

(38) Li, X.; Li, H.; Yang, D. Determination of Multiphase Boundaries and Swelling Factors of Solvent(s)- $\mathrm{CO}_{2}$-Heavy Oil Systems at High Pressures and Elevated Temperatures. Energy Fuels 2013, 27 (3), 1293-1306.

(39) Li, H.; Yang, D.; Li, X. Determination of Three-Phase Boundaries of Solvent(s)- $\mathrm{CO}_{2}$-Heavy Oil Systems under Reservoir Conditions. Energy Fuels 2013, 27 (1), 145-153.

(40) Li, X.; Han, H.; Yang, D.; Liu, X.; Qin, J. Phase Behavior of $\mathrm{C}_{3} \mathrm{H}_{8}-\mathrm{CO}_{2}$-Heavy Oil Systems in the Presence of Aqueous Phase under Reservoir Conditions. Fuel 2017, 209, 358-370.

(41) Li, X.; Yang, D.; Fan, Z. Vapor-Liquid Phase Boundaries and Swelling Factors of $\mathrm{C}_{3} \mathrm{H}_{8}-n-\mathrm{C}_{4} \mathrm{H}_{10}-\mathrm{CO}_{2}$-Heavy Oil Systems under Reservoir Conditions. Fluid Phase Equilib. 2017, 434, 211-221.

(42) Burgers, G.; Van Leeuwen, P.; Evensen, G. Analysis Scheme in the Ensemble Kalman Filter. Mon. Weather Rev. 1998, 126 (6), 17191724. 